Mulyava O. M., Sheremeta M. M.

\title{
RELATIVE GROWTH OF ENTIRE DIRICHLET SERIES WITH DIFFERENT GENERALIZED ORDERS
}

\begin{abstract}
For entire functions $F$ and $G$ defined by Dirichlet series with exponents increasing to $+\infty$ formulas are found for the finding the generalized order $\varrho_{\alpha, \beta}[F]_{G}=\varlimsup_{\sigma \rightarrow=\infty} \frac{\alpha\left(M_{G}^{-1}\left(M_{F}(\sigma)\right)\right)}{\beta(\sigma)}$ and the generalized lower order $\lambda_{\alpha, \beta}[F]_{G}=\varliminf_{\sigma \rightarrow+\infty} \frac{\alpha\left(M_{G}^{-1}\left(M_{F}(\sigma)\right)\right)}{\beta(\sigma)}$ of $F$ with respect to $G$, where $M_{F}(\sigma)=\sup \{|F(\sigma+i t)|: t \in \mathbb{R}\}$ and $\alpha$ and $\beta$ are positive increasing to $+\infty$ functions.

Key words and phrases: Dirichlet series, generalized order, relative growth.
\end{abstract}

Kyiv National University of Food Technologies, Kyiv, Ukraine (Mulyava O. M.)

Ivan Franko National University of Lviv, Lviv, Ukraine (Sheremeta M. M.)

e-mail: oksana.m@bigmir.net (Mulyava O. M.),m.m.sheremeta@gmail.com (Sheremeta M. M.)

\section{INTRODUCTION}

Let $f$ and $g$ be entire transcendental functions and $M_{f}(r)=\max \{|f(z)|:|z|=r\}$. For the study of relative growth of the functions $f$ and $g$ Ch. Roy [1] used the order $\varrho_{g}[f]=\varlimsup_{r \rightarrow+\infty} \ln M_{g}^{-1}\left(M_{f}(r)\right) / \ln r$ and the lower order $\lambda_{g}[f]=\varliminf_{r \rightarrow+\infty}^{\lim } \ln M_{g}^{-1}\left(M_{f}(r)\right) / \ln r$ of the function $f$ with respect to the function $g$. Researches of relative growth of entire functions was continued by S.K. Data, T. Biswas and other mathematicians (see, for example, $[2,3,4,5])$ in terms of maximal terms, Nevanlinna characteristic function and $k$-logarithmic orders. In [6] it is considered a relative growth of entire functions of two complex variables and in [7] the relative growth of entire Dirichlet series is studied in terms of $R$-orders.

Suppose that $\Lambda=\left(\lambda_{n}\right)$ is an increasing to $+\infty$ sequence of non-negative numbers, and by $S(\Lambda)$ we denote a class of entire Dirichlet series

$$
F(s)=\sum_{n=1}^{\infty} f_{n} \exp \left\{s \lambda_{n}\right\}, \quad s=\sigma+i t .
$$

For $\sigma<+\infty$ we put $M_{F}(\sigma)=\sup \{|F(\sigma+i t)|: t \in \mathbb{R}\}$. We remark that the function $M_{F}(\sigma)$ is continuous and increasing to $+\infty$ on $(-\infty,+\infty)$ and, therefore, there exists the function $M_{F}^{-1}(x)$ inverse to $M_{F}(\sigma)$, which increase to $+\infty$ on $\left(x_{0},+\infty\right)$.

УДК 517.537.72

2010 Mathematics Subject Classification: 30B50.

(C) Mulyava O. M., Sheremeta M. M., 2021 
By $L$ we denote a class of continuous non-negative on $(-\infty,+\infty)$ functions $\alpha$ such that $\alpha(x)=\alpha\left(x_{0}\right) \geq 0$ for $x \leq x_{0}$ and $\alpha(x) \uparrow+\infty$ as $x_{0} \leq x \rightarrow+\infty$. We say that $\alpha \in L^{0}$, if $\alpha \in L$ and $\alpha((1+o(1)) x)=(1+o(1)) \alpha(x)$ as $x \rightarrow+\infty$. Finally, $\alpha \in L_{s i}$, if $\alpha \in L$ and $\alpha(c x)=(1+o(1)) \alpha(x)$ as $x \rightarrow+\infty$ for each $c \in(0,+\infty)$, i. e. $\alpha$ is a slowly increasing function. Clearly, $L_{s i} \subset L^{0}$.

If $\alpha \in L, \beta \in L$ and $F \in S(\Lambda,+\infty)$ then the quantities

$$
\varrho_{\alpha, \beta}[F]=\varlimsup_{\sigma \rightarrow+\infty} \alpha\left(\ln M_{F}(\sigma)\right) / \beta(\sigma), \quad \lambda_{\alpha, \beta}[F]=\varliminf_{\sigma \rightarrow+\infty} \alpha\left(\ln M_{F}(\sigma)\right) / \beta(\sigma)
$$

are called [8] the generalized $(\alpha, \beta)$-order and the generalized lower $(\alpha, \beta)$-order of $F$ accordingly. We say that $F$ has the generalized regular $(\alpha, \beta)$-growth, if $0<\lambda_{\alpha, \beta}[F]=\varrho_{\alpha, \beta}[F]<$ $+\infty$.

We define the generalized $(\alpha, \beta)$-order $\varrho_{\alpha, \beta}[F]_{G}$ and the generalized lower $(\alpha, \beta)$-order $\lambda_{\alpha, \beta}[F]_{G}$ of the function $F \in S(\Lambda)$ with respect to a function $G \in S(\Lambda)$, given by Dirichlet series $G(s)=\sum_{n=1}^{\infty} g_{n} \exp \left\{s \lambda_{n}\right\}$, as follows

$$
\varrho_{\alpha, \beta}[F]_{G}=\varlimsup_{\sigma \rightarrow+\infty} \alpha\left(M_{G}^{-1}\left(M_{F}(\sigma)\right)\right) / \beta(\sigma), \quad \lambda_{\alpha, \beta}[F]_{G}=\varliminf_{\sigma \rightarrow+\infty} \alpha\left(M_{G}^{-1}\left(M_{F}(\sigma)\right)\right) / \beta(\sigma) .
$$

The following theorems are proved in [9].

Theorem (A). Let $\beta \in L$ and $\gamma \in L$. Except for the cases, when $\varrho_{\gamma, \beta}[F]=\varrho_{\gamma, \beta}[G]=0$ or $\varrho_{\gamma, \beta}[F]=\varrho_{\gamma, \beta}[G]=+\infty$, the inequality $\varrho_{\beta, \beta}[F]_{G} \geq \varrho_{\gamma, \beta}[F] / \varrho_{\gamma, \beta}[G]$ is true and subject to the condition of the generalized regular $(\gamma, \beta)$-growth of $G$ this inequality converts into an equality.

Except for the cases, when $\lambda_{\gamma, \beta}[F]=\lambda_{\gamma, \beta}[G]=0$ or $\lambda_{\gamma, \beta}[F]=\lambda_{\gamma, \beta}[G]=+\infty$, the inequality $\lambda_{\beta, \beta}[F]_{G} \leq \lambda_{\gamma, \beta}[F] / \lambda_{\gamma, \beta}[G]$ is true and subject to the condition of the generalized regular $(\gamma, \beta)$-growth of $G$ this inequality converts into an equality.

Theorem (B). Let $0<p<+\infty$ and one of conditions is executed:

a) $\gamma \in L^{0}, \beta(\ln x) \in L^{0}, \frac{d \beta^{-1}(c \gamma(x))}{d \ln x} \rightarrow \frac{1}{p}(x \rightarrow+\infty)$ for each $c \in(0,+\infty)$ and $\ln n=o\left(\lambda_{n}\right)(n \rightarrow \infty)$;

b) $\gamma \in L_{s i}, \beta \in L^{0}, \varrho_{\gamma, \beta}[F]<+\infty, \frac{d \beta^{-1}(c \gamma(x))}{d \ln x}=O(1)(x \rightarrow+\infty)$ and $\ln n=$ $=o\left(\lambda_{n} \beta^{-1}\left(c \gamma\left(\lambda_{n}\right)\right)\right)(n \rightarrow \infty)$ for each $c \in(0,+\infty)$.

Suppose that $\gamma\left(\lambda_{n+1} / p\right)=(1+o(1)) \gamma\left(\lambda_{n} / p\right)$ as $n \rightarrow \infty$.

If the function $G$ has generalized regular $(\gamma, \beta)$-growth and $\kappa_{n}[G]:=\frac{\ln \left|g_{n}\right|-\ln \left|g_{n+1}\right|}{\lambda_{n+1}-\lambda_{n}} \nearrow$ $+\infty$ as $n_{0} \leq n \rightarrow \infty$ then

$$
\varrho_{\beta, \beta}[F]_{G}=\varlimsup_{n \rightarrow \infty} \beta\left(\frac{1}{p}+\frac{1}{\lambda_{n}} \ln \frac{1}{\left|g_{n}\right|}\right) / \beta\left(\frac{1}{p}+\frac{1}{\lambda_{n}} \ln \frac{1}{\left|f_{n}\right|}\right)
$$

except for the cases, when $\varrho_{\gamma, \beta}[F]=\varrho_{\gamma, \beta}[G]=0$ or $\varrho_{\gamma, \beta}[F]=\varrho_{\gamma, \beta}[G]=+\infty$.

If, moreover, $\kappa_{n}[F] \nearrow+\infty$ as $n_{0} \leq n \rightarrow \infty$ then

$$
\lambda_{\beta, \beta}[F]_{G}=\varliminf_{n \rightarrow \infty} \beta\left(\frac{1}{p}+\frac{1}{\lambda_{n}} \ln \frac{1}{\left|g_{n}\right|}\right) / \beta\left(\frac{1}{p}+\frac{1}{\lambda_{n}} \ln \frac{1}{\left|f_{n}\right|}\right)
$$


except for the cases, when $\lambda_{\gamma, \beta}[F]=\lambda_{\gamma, \beta}[G]=0$ or $\lambda_{\gamma, \beta}[F]=\lambda_{\gamma, \beta}[G]=+\infty$.

Similar results in terms of $R$-types are obtained in [10].

Here we consider the general case when $\alpha \neq \beta$.

\section{Analogues of Theorem (A)}

We begin from the following general theorem.

Theorem 1. If $\alpha \in L$ and $\beta \in L$ then:

1) the inequalities

$$
\frac{\varrho_{\gamma, \beta}[F]}{\varrho_{\gamma, \alpha}[G]} \leq \varrho_{\alpha, \beta}[F]_{G} \leq \frac{\varrho_{\gamma, \beta}[F]}{\lambda_{\gamma, \alpha}[G]}
$$

are true for each function $\gamma \in L$ except for the cases $\varrho_{\gamma, \beta}[F]=\varrho_{\gamma, \alpha}[G]=0$, $\varrho_{\gamma, \beta}[F]=\lambda_{\gamma, \alpha}[G]=0, \varrho_{\gamma, \beta}[F]=\varrho_{\gamma, \alpha}[G]=+\infty, \varrho_{\gamma, \beta}[F]=\lambda_{\gamma, \alpha}[G]=+\infty$;

2) the inequalities

$$
\frac{\lambda_{\gamma, \beta}[F]}{\varrho_{\gamma, \alpha}[G]} \leq \lambda_{\alpha, \beta}[F]_{G} \leq \frac{\lambda_{\gamma, \beta}[F]}{\lambda_{\gamma, \alpha}[G]}
$$

are true for each function $\gamma \in L$ except for the cases $\lambda_{\gamma, \beta}[F]=\lambda_{\gamma, \alpha}[G]=0$, $\lambda_{\gamma, \beta}[F]=\varrho_{\gamma, \alpha}[G]=0, \lambda_{\gamma, \beta}[F]=\lambda_{\gamma, \alpha}[G]=+\infty, \lambda_{\gamma, \beta}[F]=\varrho_{\gamma, \alpha}[G]=+\infty$.

Proof. Indeed,

$$
\begin{gathered}
\varrho_{\alpha, \beta}[F]_{G}=\varlimsup_{x \rightarrow+\infty} \frac{\alpha\left(M_{G}^{-1}(x)\right)}{\beta\left(M_{F}^{-1}(x)\right)}=\varlimsup_{x \rightarrow+\infty} \frac{\gamma(\ln x)}{\beta\left(M_{F}^{-1}(x)\right)} \frac{\alpha\left(M_{G}^{-1}(x)\right)}{\gamma(\ln x)} \geq \\
\geq \varlimsup_{x \rightarrow+\infty} \frac{\gamma(\ln x)}{\beta\left(M_{F}^{-1}(x)\right)} \varliminf_{x \rightarrow+\infty} \frac{\alpha\left(M_{G}^{-1}(x)\right)}{\gamma(\ln x)}=\varlimsup_{\sigma \rightarrow+\infty} \frac{\left.\gamma\left(\ln M_{F}(\sigma)\right)\right)}{\beta(\sigma)} \varliminf_{\sigma \rightarrow+\infty} \frac{\alpha(\sigma)}{\gamma\left(\ln M_{G}(\sigma)\right)}=\frac{\varrho_{\gamma, \beta}[F]}{\varrho_{\gamma, \alpha}[G]}
\end{gathered}
$$

and

$$
\begin{gathered}
\varrho_{\alpha, \beta}[F]_{G} \leq \varlimsup_{x \rightarrow+\infty} \frac{\gamma(\ln x)}{\beta\left(M_{F}^{-1}(x)\right)} \varlimsup_{x \rightarrow+\infty} \frac{\alpha\left(M_{G}^{-1}(x)\right)}{\gamma(\ln x)}=\varlimsup_{\sigma \rightarrow+\infty} \frac{\left.\gamma\left(\ln M_{F}(\sigma)\right)\right)}{\beta(\sigma)} \varlimsup_{\sigma \rightarrow+\infty} \frac{\alpha(\sigma)}{\gamma\left(\ln M_{G}(\sigma)\right)}= \\
=\frac{\varrho_{\gamma, \beta}[F]}{\lambda_{\gamma, \alpha}[G]},
\end{gathered}
$$

i. e. inequalities (2) are proved.

The proof of (3) is similar. Indeed,

$$
\lambda_{\alpha, \beta}[F]_{G}=\lim _{x \rightarrow+\infty} \frac{\gamma(\ln x)}{\beta\left(M_{F}^{-1}(x)\right)} \frac{\alpha\left(M_{G}^{-1}(x)\right)}{\gamma(\ln x)} \leq \lim _{x \rightarrow+\infty} \frac{\gamma(\ln x)}{\beta\left(M_{F}^{-1}(x)\right)} \varlimsup_{x \rightarrow+\infty} \frac{\alpha\left(M_{G}^{-1}(x)\right)}{\gamma(\ln x)}=\frac{\lambda_{\gamma, \beta}[F]}{\lambda_{\gamma, \alpha}[G]}
$$

and

$$
\lambda_{\alpha, \beta}[F]_{G} \geq \varliminf_{x \rightarrow+\infty} \frac{\gamma(\ln x)}{\beta\left(M_{F}^{-1}(x)\right)} \varliminf_{x \rightarrow+\infty} \frac{\alpha\left(M_{G}^{-1}(x)\right)}{\gamma(\ln x)}=\frac{\lambda_{\gamma, \beta}[F]}{\varrho_{\gamma, \alpha}[G]},
$$

whence (3) follows. Theorem 1 is proved. 
Remark 1. In the statements 1) and 2) of Theorem 1 the conditions for the function $\gamma$ hold if $0<\lambda_{\gamma, \alpha}[G] \leq \varrho_{\gamma, \alpha}[G]<+\infty$. From (2) and (3) it follows that if $G$ has the generalized regular $(\gamma, \alpha)$-growth then $\varrho_{\alpha, \beta}[F]_{G}=\varrho_{\gamma, \beta}[F] / \varrho_{\gamma, \alpha}[G]$.

If we choose $\alpha(x)=\ln x$ and $\beta(x)=x$ for $x \geq 3$ then from the definition of $\varrho_{\gamma, \beta}[F]$ and $\lambda_{\gamma, \beta}[F]$ we obtain the definition of the $R$-order $\varrho_{R}[G]$ and the lower $R$-order $\lambda_{R}[G]$ introduced by J. Ritt [11], and if we choose $\alpha(x)=\beta(x)=\ln x$ for $x \geq 3$ then we obtain the definition of the logarithmic order $\varrho_{l}[G]$ and the lower logarithmic order $\lambda_{l}[G]$.

For the characteristic of the relative growth of the function $F$ with respect to a function $G$ in Ritt's scale we use

$$
\varrho_{R, R}[F]_{G}=\varlimsup_{\sigma \rightarrow+\infty} M_{G}^{-1}\left(M_{F}(\sigma)\right) / \sigma, \quad \lambda_{R, R}[F]_{G}=\varliminf_{\sigma \rightarrow+\infty}^{\varliminf_{G}} M_{G}^{-1}\left(M_{F}(\sigma)\right) / \sigma,
$$

in the logarithmic scale we use

$$
\varrho_{l, l}[F]_{G}=\varlimsup_{\sigma \rightarrow+\infty} \ln M_{G}^{-1}\left(M_{F}(\sigma)\right) / \ln \sigma, \quad \lambda_{l, l}[F]_{G}=\varliminf_{\sigma \rightarrow+\infty} \ln M_{G}^{-1}\left(M_{F}(\sigma)\right) / \ln \sigma
$$

and in the mixed scale we use

$$
\varrho_{R, l}[F]_{G}=\varlimsup_{\sigma \rightarrow+\infty} \ln M_{G}^{-1}\left(M_{F}(\sigma)\right) / \sigma, \quad \lambda_{R, l}[F]_{G}=\underset{\sigma \rightarrow+\infty}{\lim } \ln M_{G}^{-1}\left(M_{F}(\sigma)\right) / \sigma .
$$

Then Theorem 1 implies the following statement.

Corollary 1. If $0<\lambda_{R}[G] \leq \varrho_{R}[G]<+\infty$ then

$$
\frac{\varrho_{R}[F]}{\varrho_{R}[G]} \leq \varrho_{R, R}[F]_{G} \leq \frac{\varrho_{R}[F]}{\lambda_{R}[G]} \quad \text { and } \quad \frac{\lambda_{R}[F]}{\varrho_{R}[G]} \leq \lambda_{R, R}[F]_{G} \leq \frac{\lambda_{R}[F]}{\lambda_{R}[G]}
$$

If $0<\lambda_{l}[G] \leq \varrho_{l}[G]<+\infty$ then

$$
\frac{\varrho_{l}[F]}{\varrho_{l}[G]} \leq \varrho_{l, l}[F]_{G} \leq \frac{\varrho_{l}[F]}{\lambda_{l}[G]} \quad \text { and } \quad \frac{\lambda_{l}[F]}{\varrho_{l}[G]} \leq \lambda_{l, l}[F]_{G} \leq \frac{\lambda_{l}[F]}{\lambda_{l}[G]}
$$

If $0<\lambda_{l}[G] \leq \varrho_{l}[G]<+\infty$ then

$$
\frac{\varrho_{R}[F]}{\varrho_{l}[G]} \leq \varrho_{R, l}[F]_{G} \leq \frac{\varrho_{R}[F]}{\lambda_{l}[G]} \quad \text { and } \quad \frac{\lambda_{R}[F]}{\varrho_{l}[G]} \leq \lambda_{R, l}[F]_{G} \leq \frac{\lambda_{R}[F]}{\lambda_{l}[G]}
$$

For a more detailed description of the growth of Dirichlet series of finite nonzero order use the type. If $0<\varrho_{R}[F]<+\infty$ then the quantities

$$
T_{R}[F]=\varlimsup_{\sigma \rightarrow+\infty} \frac{\ln M_{F}(\sigma)}{\exp \left\{\sigma \varrho_{R}[F]\right\}} \quad \text { and } \quad t_{R}[F]=\varliminf_{\sigma \rightarrow+\infty} \frac{\ln M_{F}(\sigma)}{\exp \left\{\sigma \varrho_{R}[F]\right\}}
$$

are called the $R$-type and the lower $R$-type of function $F$. Similarly, the quantities

$$
T_{l}[F]=\varlimsup_{\sigma \rightarrow+\infty} \frac{\ln M_{F}(\sigma)}{\sigma^{\varrho_{l}}[F]} \quad \text { and } \quad t_{l}[F]=\varliminf_{\sigma \rightarrow+\infty} \frac{\ln M_{F}(\sigma)}{\sigma^{\varrho_{l}}[F]}
$$


are called the logarithmic type and the lower logarithmic type of function $F$. Therefore, by analogy, if $0<\varrho_{\alpha, \beta}[F]<+\infty$ then we define the generalized $(\alpha, \beta)$-type and the lower generalized $(\alpha, \beta)$-type of $F$ as follows

$$
T_{\alpha, \beta}[F]=\varlimsup_{\sigma \rightarrow+\infty} \frac{\exp \left\{\alpha\left(\ln M_{F}(\sigma)\right)\right\}}{\exp \left\{\beta(\sigma) \varrho_{\alpha, \beta}[F]\right\}}, \quad t_{\alpha, \beta}[F]=\varliminf_{\sigma \rightarrow+\infty} \frac{\exp \left\{\alpha\left(\ln M_{F}(\sigma)\right)\right\}}{\exp \left\{\beta(\sigma) \varrho_{\alpha, \beta}[F]\right\}} .
$$

Similarly, if $0<\varrho_{\alpha, \beta}[F]_{G}<+\infty$ then we define the generalized $(\alpha, \beta)$-type and the lower generalized $(\alpha, \beta)$-type of the function $F$ with respect to the function $G$ as follows

$$
T_{\alpha, \beta}[F]_{G}=\varlimsup_{\sigma \rightarrow+\infty} \frac{\exp \left\{\alpha\left(M_{G}^{-1}\left(M_{F}(\sigma)\right)\right)\right\}}{\exp \left\{\beta(\sigma) \varrho_{\alpha, \beta}[F]_{G}\right\}}, \quad t_{\alpha, \beta}[F]_{G}=\varliminf_{\sigma \rightarrow+\infty} \frac{\exp \left\{\alpha\left(M_{G}^{-1}\left(M_{F}(\sigma)\right)\right)\right\}}{\exp \left\{\beta(\sigma) \varrho_{\alpha, \beta}[F]_{G}\right\}} .
$$

Theorem 2. Let $\alpha \in L, \beta \in L$ and $\gamma \in L$. If the function $G$ has the regular generalized $(\gamma, \alpha)$-growth and $0<t_{\gamma, \alpha}[G] \leq T_{\gamma, \alpha}[G]<+\infty$ then

$$
\frac{T_{\gamma, \beta}[F]}{T_{\gamma, \alpha}[G]} \leq\left(T_{\alpha, \beta}[F]_{G}\right)^{\varrho_{\gamma, \alpha}[G]} \leq \frac{T_{\gamma, \beta}[F]}{t_{\gamma, \alpha}[G]}
$$

and

$$
\frac{t_{\gamma, \beta}[F]}{T_{\gamma, \alpha}[G]} \leq\left(t_{\alpha, \beta}[F]_{G}\right)^{\varrho_{\gamma, \alpha}[G]} \leq \frac{t_{\gamma, \beta}[F]}{t_{\gamma, \alpha}[G]}
$$

Proof. Since $G$ has the regular generalized $(\gamma, \alpha)$-growth, by Theorem 1 (see Remark 1) we have $\varrho_{\alpha, \beta}[F]_{G}=\frac{\varrho_{\gamma, \beta}[F]}{\varrho_{\gamma, \alpha}[G]}$. Therefore,

$$
\begin{aligned}
\left(T_{\alpha, \beta}[F]_{G}\right)^{\varrho_{\gamma, \alpha}[G]} & =\varlimsup_{\sigma \rightarrow+\infty} \frac{\exp \left\{\varrho_{\gamma, \alpha}[G] \alpha\left(M_{G}^{-1}\left(M_{F}(\sigma)\right)\right)\right\}}{\exp \left\{\beta(\sigma) \varrho_{\gamma, \beta}[F]\right\}}=\varlimsup_{x \rightarrow+\infty} \frac{\exp \left\{\varrho_{\gamma, \alpha}[G] \alpha\left(M_{G}^{-1}(x)\right)\right\}}{\exp \left\{\varrho_{\gamma, \beta}[F] \beta\left(M_{F}^{-1}(x)\right)\right\}}= \\
& =\varlimsup_{x \rightarrow+\infty} \frac{\exp \{\gamma(\ln x)\}}{\exp \left\{\varrho_{\gamma, \beta}[F] \beta\left(M_{F}^{-1}(x)\right)\right\}} \frac{\exp \left\{\varrho_{\gamma, \alpha}[G] \alpha\left(M_{G}^{-1}(x)\right)\right\}}{\exp \{\gamma(\ln x)\}} \geq \\
\geq & \varlimsup_{x \rightarrow+\infty} \frac{\exp \{\gamma(\ln x)\}}{\exp \left\{\varrho_{\gamma, \beta}[F] \beta\left(M_{F}^{-1}(x)\right)\right\}} \underset{x \rightarrow+\infty}{\lim _{x \rightarrow+\infty}} \frac{\exp \left\{\varrho_{\gamma, \alpha}[G] \alpha\left(M_{G}^{-1}(x)\right)\right\}}{\exp \{\gamma(\ln x)\}}= \\
& =\varlimsup_{\sigma \rightarrow+\infty} \frac{\exp \left\{\gamma\left(\ln M_{F}(\sigma)\right)\right\}}{\exp \left\{\varrho_{\gamma, \beta}[F] \beta(\sigma)\right\}} \underset{\sigma \rightarrow+\infty}{\lim } \frac{\exp \left\{\varrho_{\gamma, \alpha}[G] \alpha(\sigma)\right\}}{\exp \left\{\gamma\left(\ln M_{G}(\sigma)\right)\right\}}=\frac{T_{\gamma, \beta}[F]}{T_{\gamma, \alpha}[G]}
\end{aligned}
$$

and

$$
\begin{gathered}
\left(T_{\alpha, \beta}[F]_{G}\right)^{\varrho_{\gamma, \alpha}[G]} \leq \varlimsup_{x \rightarrow+\infty} \frac{\exp \{\gamma(\ln x)\}}{\exp \left\{\varrho_{\gamma, \beta}[F] \beta\left(M_{F}^{-1}(x)\right)\right\}} \varlimsup_{x \rightarrow+\infty} \frac{\exp \left\{\varrho_{\gamma, \alpha}[G] \alpha\left(M_{G}^{-1}(x)\right)\right\}}{\exp \{\gamma(\ln x)\}}= \\
=\varlimsup_{\sigma \rightarrow+\infty} \frac{\exp \left\{\gamma\left(\ln M_{F}(\sigma)\right)\right\}}{\exp \left\{\varrho_{\gamma, \beta}[F] \beta(\sigma)\right\}} \varlimsup_{\sigma \rightarrow+\infty} \frac{\exp \left\{\varrho_{\gamma, \alpha}[G] \alpha(\sigma)\right\}}{\exp \left\{\gamma\left(\ln M_{G}(\sigma)\right)\right\}}=\frac{T_{\gamma, \beta}[F]}{t_{\gamma, \alpha}[G]} .
\end{gathered}
$$

Estimates (4) are proved. The proof of (5) is similar and we will omit it.

Theorem 2 implies the following statement. 
Corollary 2. If the function $G$ has the regular growth and $0<t_{R}[G] \leq T_{R}[G]<+\infty$ then

$$
\frac{T_{R}[F]}{T_{R}[G]} \leq\left(T_{R, R}[F]_{G}\right)^{\varrho_{R}[G]} \leq \frac{T_{R}[F]}{t_{R}[G]}, \quad \frac{t_{R}[F]}{T_{R}[G]} \leq\left(t_{R, R}[F]_{G}\right)^{\varrho_{R}[G]} \leq \frac{t_{R}[F]}{t_{R}[G]}
$$

where

$$
T_{R, R}[F]_{G}=\varlimsup_{\sigma \rightarrow+\infty} \frac{\exp \left\{M_{G}^{-1}\left(M_{F}(\sigma)\right)\right\}}{\exp \left\{\sigma \varrho_{R, R}[F]_{G}\right\}}, \quad t_{R, R}[F]_{G}=\varliminf_{\sigma \rightarrow+\infty} \frac{\exp \left\{M_{G}^{-1}\left(M_{F}(\sigma)\right)\right\}}{\exp \left\{\sigma \varrho_{R, R}[F]_{G}\right\}} .
$$

If the function $G$ has the regular logarithmic growth and $0<t_{l}[G] \leq T_{l}[G]<+\infty$ then

$$
\frac{T_{l}[F]}{T_{l}[G]} \leq\left(T_{l, l}[F]_{G}\right)^{\varrho_{l}[G]} \leq \frac{T_{l}[F]}{t_{l}[G]}, \quad \frac{t_{l}[F]}{T_{l}[G]} \leq\left(t_{l, l}[F]_{G}\right)^{\varrho_{l}[G]} \leq \frac{t_{l}[F]}{t_{l}[G]}
$$

where

$$
T_{l, l}[F]_{G}=\varlimsup_{\sigma \rightarrow+\infty} \sigma^{-\varrho_{l, l}[F]_{G}} M_{G}^{-1}\left(M_{F}(\sigma)\right), \quad t_{l, l}[F]_{G}=\underline{\lim }_{\sigma \rightarrow+\infty} \sigma^{-\varrho_{l, l}[F]_{G}} M_{G}^{-1}\left(M_{F}(\sigma)\right) .
$$

If the function $G$ has the regular logarithmic growth and $0<t_{l}[G] \leq T_{l}[G]<+\infty$ then

$$
\frac{T_{R}[F]}{T_{l}[G]} \leq\left(T_{R, l}[F]_{G}\right)^{\varrho_{l}[G]} \leq \frac{T_{R}[F]}{t_{l}[G]}, \quad \frac{t_{R}[F]}{T_{l}[G]} \leq\left(t_{R, l}[F]_{G}\right)^{\varrho_{l}[G]} \leq \frac{t_{R}[F]}{t_{l}[G]}
$$

where

$$
T_{R, l}[F]_{G}:=\varlimsup_{\sigma \rightarrow+\infty} \frac{M_{G}^{-1}\left(M_{F}(\sigma)\right)}{\exp \left\{\sigma \varrho_{R, l}[F]_{G}\right\}}, \quad t_{R, l}[F]_{G}:=\varliminf_{\sigma \rightarrow+\infty} \frac{M_{G}^{-1}\left(M_{F}(\sigma)\right)}{\exp \left\{\sigma \varrho_{R, l}[F]_{G}\right\}} .
$$

\section{Analogues of Theorem (B)}

We need the following lemma.

Lemma 1 ([8]). Let $\alpha \in L_{s i}, \beta \in L^{0}$ and $\frac{d \beta^{-1}(c \alpha(x))}{d \ln x}=O(1)$ as $x \rightarrow+\infty$ for each $c \in(0,+\infty)$. If $\ln n=o\left(\lambda_{n} \beta^{-1}\left(c \alpha\left(\lambda_{n}\right)\right)\right)$ as $n \rightarrow \infty$ for each $c \in(0,+\infty)$ and $G \in S(\Lambda)$ then

$$
\varrho_{\alpha, \beta}[G]=\varlimsup_{n \rightarrow \infty} \alpha\left(\lambda_{n}\right) / \beta\left(\frac{1}{\lambda_{n}} \ln \frac{1}{\left|g_{n}\right|}\right) .
$$

If, moreover, $\alpha\left(\lambda_{n+1}\right) \sim \alpha\left(\lambda_{n}\right)$ and $\kappa_{n}[G] \nearrow+\infty$ as $n_{0} \leq n \rightarrow \infty$ then

$$
\lambda_{\alpha, \beta}[G]=\varliminf_{n \rightarrow \infty} \alpha\left(\lambda_{n}\right) / \beta\left(\frac{1}{\lambda_{n}} \ln \frac{1}{\left|g_{n}\right|}\right) .
$$

Now we prove the following theorem.

Theorem 3. Let $\alpha \in L^{0}, \beta \in L^{0}, \gamma \in L_{s i}, \frac{d \alpha^{-1}(c \gamma(x))}{d \ln x}=O(1)$ and $\frac{d \beta^{-1}(c \gamma(x))}{d \ln x}=O(1)$ as $x \rightarrow+\infty$ for each $c \in(0,+\infty)$. Suppose that $\ln n=o\left(\lambda_{n} \alpha^{-1}\left(c \gamma\left(\lambda_{n}\right)\right)\right)$ and $\ln n=$ $=o\left(\lambda_{n} \beta^{-1}\left(c \gamma\left(\lambda_{n}\right)\right)\right)$ as $n \rightarrow \infty$ for each $c \in(0,+\infty)$. 
If the function $G$ has generalized regular $(\gamma, \alpha)$-growth, $\gamma\left(\lambda_{n+1}\right) \sim \gamma\left(\lambda_{n}\right)$ and $\kappa_{n}[G] \nearrow+\infty$ as $n_{0} \leq n \rightarrow \infty$ then

$$
\varrho_{\alpha, \beta}[F]_{G}=P_{\alpha, \beta}:=\varlimsup_{n \rightarrow \infty} \alpha\left(\frac{1}{\lambda_{n}} \ln \frac{1}{\left|g_{n}\right|}\right) / \beta\left(\frac{1}{\lambda_{n}} \ln \frac{1}{\left|f_{n}\right|}\right) .
$$

If, moreover, $\kappa_{n}[F] \nearrow+\infty$ as $n_{0} \leq n \rightarrow \infty$ then

$$
\lambda_{\alpha, \beta}[F]_{G}=p_{\alpha, \beta}:=\varliminf_{n \rightarrow \infty} \alpha\left(\frac{1}{\lambda_{n}} \ln \frac{1}{\left|g_{n}\right|}\right) / \beta\left(\frac{1}{\lambda_{n}} \ln \frac{1}{\left|f_{n}\right|}\right) .
$$

Proof. Since $G$ has generalized regular $(\gamma, \alpha)$-growth, by Theorem $1 \varrho_{\alpha, \beta}[F]_{G}=\frac{\varrho_{\gamma, \beta}[F]}{\varrho_{\gamma, \alpha}[G]}$, $\lambda_{\alpha, \beta}[F]_{G}=\frac{\lambda_{\gamma, \beta}[F]}{\lambda_{\gamma, \alpha}[G]}$ and by Lemma 1

$$
\varrho_{\gamma, \beta}[F]=\varlimsup_{n \rightarrow \infty} \gamma\left(\lambda_{n}\right) / \beta\left(\frac{1}{\lambda_{n}} \ln \frac{1}{\left|f_{n}\right|}\right), \quad \varrho_{\gamma, \alpha}[G]=\varlimsup_{n \rightarrow \infty} \gamma\left(\lambda_{n}\right) / \alpha\left(\frac{1}{\lambda_{n}} \ln \frac{1}{\left|g_{n}\right|}\right) .
$$

Therefore,

$$
\begin{gathered}
\varrho_{\alpha, \beta}[F]_{G}=\varlimsup_{n \rightarrow \infty} \gamma\left(\lambda_{n}\right) / \beta\left(\frac{1}{\lambda_{n}} \ln \frac{1}{\left|f_{n}\right|}\right) \varliminf_{n \rightarrow \infty} \alpha\left(\frac{1}{\lambda_{n}} \ln \frac{1}{\left|g_{n}\right|}\right) / \gamma\left(\lambda_{n}\right) \leq \\
\leq \varlimsup_{n \rightarrow \infty}\left(\gamma\left(\lambda_{n}\right) / \beta\left(\frac{1}{\lambda_{n}} \ln \frac{1}{\left|f_{n}\right|}\right)\right)\left(\alpha\left(\frac{1}{\lambda_{n}} \ln \frac{1}{\left|g_{n}\right|}\right) / \gamma\left(\lambda_{n}\right)\right)=P_{\alpha, \beta} .
\end{gathered}
$$

On the other hand, let $P_{\alpha, \beta}>0$. Then for every $\varepsilon \in\left(0, P_{\alpha, \beta}\right)$ there exists an increasing to $+\infty$ sequence $\left(n_{k}\right)$ of integers such that

$$
\alpha\left(\frac{1}{\lambda_{n_{k}}} \ln \frac{1}{\left|g_{n_{k}}\right|}\right)>\left(P_{\alpha, \beta}-\varepsilon\right) \beta\left(\frac{1}{\lambda_{n_{k}}} \ln \frac{1}{\left|f_{n_{k}}\right|}\right)
$$

i. e.

$$
\gamma\left(\lambda_{n_{k}}\right) / \beta\left(\frac{1}{\lambda_{n_{k}}} \ln \frac{1}{\left|f_{n_{k}}\right|}\right)>\left(P_{\alpha, \beta}-\varepsilon\right) \gamma\left(\lambda_{n_{k}}\right) / \alpha\left(\frac{1}{\lambda_{n_{k}}} \ln \frac{1}{\left|g_{n_{k}}\right|}\right)
$$

and, thus,

$$
\begin{gathered}
\varrho_{\gamma, \beta}[F]=\varlimsup_{n \rightarrow \infty} \gamma\left(\lambda_{n}\right) / \beta\left(\frac{1}{\lambda_{n}} \ln \frac{1}{\left|f_{n}\right|}\right) \geq\left(P_{\alpha, \beta}-\varepsilon\right) \varliminf_{n \rightarrow \infty} \gamma\left(\lambda_{n}\right) / \alpha\left(\frac{1}{\lambda_{n}} \ln \frac{1}{\left|g_{n}\right|}\right)= \\
=\left(P_{\alpha, \beta}-\varepsilon\right) \lambda_{\gamma, \alpha}[G]=\left(P_{\alpha, \beta}-\varepsilon\right) \varrho_{\gamma, \alpha}[G],
\end{gathered}
$$

whence in view of the arbitrariness of $\varepsilon$ we get $\varrho_{\alpha, \beta}[F]_{G} \geq P_{\alpha, \beta}$. For $P_{\alpha, \beta}=0$ the last inequality is obvious. Equality (8) is proved.

For the proof of (9) we remark that since $G$ has generalized regular $(\alpha, \beta)$-growth, by Theorem 1 and Lemma 1

$$
\lambda_{\alpha, \beta}[F]_{G}=\varliminf_{n \rightarrow \infty} \gamma\left(\lambda_{n}\right) / \beta\left(\frac{1}{\lambda_{n}} \ln \frac{1}{\left|f_{n}\right|}\right) \varlimsup_{n \rightarrow \infty} \alpha\left(\frac{1}{\lambda_{n}} \ln \frac{1}{\left|g_{n}\right|}\right) / \gamma\left(\lambda_{n}\right) \geq
$$




$$
\geq \varliminf_{n \rightarrow \infty}\left(\gamma\left(\lambda_{n}\right) / \beta\left(\frac{1}{\lambda_{n}} \ln \frac{1}{\left|f_{n}\right|}\right)\right)\left(\alpha\left(\frac{1}{\lambda_{n}} \ln \frac{1}{\left|g_{n}\right|}\right) / \gamma\left(\lambda_{n}\right)\right)=p_{\alpha, \beta} .
$$

On the other hand, let $p_{\alpha, \beta}<+\infty$. Then for every $\varepsilon>0$ there exists an increasing to $+\infty$ sequence $\left(n_{k}\right)$ of integers such that

$$
\alpha\left(\frac{1}{\lambda_{n_{k}}} \ln \frac{1}{\left|g_{n_{k}}\right|}\right)<\left(p_{\alpha, \beta}+\varepsilon\right) \beta\left(\frac{1}{\lambda_{n_{k}}} \ln \frac{1}{\left|f_{n_{k}}\right|}\right)
$$

and, as above,

$$
\begin{gathered}
\lambda_{\gamma, \beta}[F]=\varliminf_{n \rightarrow \infty} \gamma\left(\lambda_{n}\right) / \beta\left(\frac{1}{\lambda_{n}} \ln \frac{1}{\left|f_{n}\right|}\right) \leq\left(p_{\alpha, \beta}+\varepsilon\right) \varlimsup_{n \rightarrow \infty} \gamma\left(\lambda_{n}\right) / \alpha\left(\frac{1}{\lambda_{n}} \ln \frac{1}{\left|g_{n}\right|}\right)= \\
=\left(p_{\alpha, \beta}+\varepsilon\right) \varrho_{\gamma, \alpha}[G]=\left(p_{\alpha, \beta}+\varepsilon\right) \lambda_{\gamma, \alpha}[G],
\end{gathered}
$$

whence in view of the arbitrariness of $\varepsilon$ we get $\lambda_{\alpha, \beta}[F]_{G} \leq p_{\alpha, \beta}$. For $p_{\alpha, \beta}=+\infty$ the last inequality is obvious. Equality (9) is proved, and the proof of Theorem 3 is complete.

For the study of the relative growth in classical scales we need the following lemmas.

Lemma 2 ([11], [12], [13], [14]). If $\ln n=o\left(\lambda_{n} \ln \lambda_{n}\right)$ as $n \rightarrow \infty$ then

$$
\varrho_{R}[F]=\varlimsup_{n \rightarrow \infty} \lambda_{n} \ln \lambda_{n} /\left(-\ln \left|f_{n}\right|\right)
$$

and if, moreover, $\ln \lambda_{n+1} \sim \ln \lambda_{n}$ and $\kappa_{n}[G] \nearrow+\infty$ as $n_{0} \leq n \rightarrow \infty$ then

$$
\lambda_{R}[F]=\varliminf_{n \rightarrow \infty} \lambda_{n} \ln \lambda_{n} /\left(-\ln \left|f_{n}\right|\right) \text {. }
$$

If $\ln n=o\left(\lambda_{n}\right)$ as $n \rightarrow \infty$ then

$$
T_{R}[F]=\left(1 /\left(e \varrho_{R}[F]\right)\right) \varlimsup_{n \rightarrow \infty} \lambda_{n}\left|f_{n}\right|^{\varrho_{R}[F] / \lambda_{n}}
$$

and if, moreover, $\lambda_{n+1} \sim \lambda_{n}$ and $\kappa_{n}[G] \nearrow+\infty$ as $n_{0} \leq n \rightarrow \infty$ then

$$
t_{R}[F]=\left(1 /\left(e \varrho_{R}[F]\right)\right) \varliminf_{n \rightarrow \infty} \lambda_{n}\left|f_{n}\right|^{\varrho_{R}[F] / \lambda_{n}} .
$$

Lemma 3 ([8]). If $\varlimsup_{n \rightarrow \infty} \ln \ln n / \ln \lambda_{n} \leq 1$ then

$$
\varrho_{l}[F]=1+\varlimsup_{n \rightarrow \infty} \ln \lambda_{n} / \ln \left(\frac{1}{\lambda_{n}} \ln \frac{1}{\left|f_{n}\right|}\right)
$$

and if, moreover, $\ln \lambda_{n+1} \sim \ln \lambda_{n}$ and $\kappa_{n}[G] \nearrow+\infty$ as $n_{0} \leq n \rightarrow \infty$ then

$$
\lambda_{l}[F]=1+\varliminf_{n \rightarrow \infty} \ln \lambda_{n} / \ln \left(\frac{1}{\lambda_{n}} \ln \frac{1}{\left|f_{n}\right|}\right) .
$$

If $1<\varrho_{l}[F]<+\infty$ and $\ln n=o\left(\lambda_{n}^{\varrho_{l}[F] /\left(\varrho_{l}[F]-1\right)}\right)$ as $n \rightarrow \infty$ then

$$
T_{l}[F]=A\left(\varrho_{l}[F]\right) \varlimsup_{n \rightarrow \infty} \lambda_{n}^{\varrho_{l}[F]}\left(\ln \frac{1}{\left|f_{n}\right|}\right)^{1-\varrho_{l}[F]}, \quad A(\varrho)=(\varrho-1)^{\varrho-1} \varrho^{\varrho},
$$

and if, moreover, $\lambda_{n+1} \sim \lambda_{n}$ and $\kappa_{n}[G] \nearrow+\infty$ as $n_{0} \leq n \rightarrow \infty$ then

$$
t_{l}[F]=A\left(\varrho_{l}[F]\right) \varliminf_{n \rightarrow \infty} \lambda_{n}^{\varrho_{l}[F]}\left(\ln \frac{1}{\left|f_{n}\right|}\right)^{1-\varrho_{l}[F]}
$$


Choosing $\alpha(x)=\beta(x)=x$ and $\gamma(x)=\ln x$, from Theorem 3 we obtain the following statement.

Proposition 1. If the function $G$ has regular growth, $\kappa_{n}[G] \nearrow+\infty, \ln n=o\left(\lambda_{n} \ln \lambda_{n}\right)$ and $\ln \lambda_{n+1} \sim \ln \lambda_{n}$ as $n_{0} \leq n \rightarrow \infty$ then $\varrho_{R, R}[F]_{G}=\varlimsup_{n \rightarrow \infty} \ln \left|g_{n}\right| / \ln \left|f_{n}\right|$.

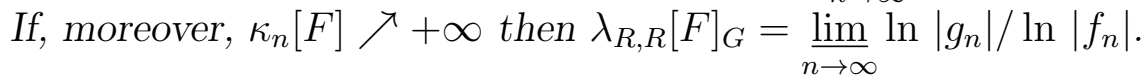

This result can be directly obtained using Lemma 2. It is easy to see also that the functions $\alpha(x)=\beta(x)=\gamma(x)=\ln x$ do not satisfy the conditions of Theorem 3. However, the following statement is correct.

Proposition 2. If the function $G$ has regular logarithmic growth, $\varlimsup_{n \rightarrow \infty} \ln \ln n / \ln \lambda_{n} \leq 1$, $\ln \lambda_{n+1} \sim \ln \lambda_{n}$ and $\kappa_{n}[G] \nearrow+\infty$ as $n_{0} \leq n \rightarrow \infty$ then

$$
\varrho_{l, l}[F]_{G}=P_{l}:=\varlimsup_{n \rightarrow \infty} \frac{\ln \ln \frac{1}{\left|f_{n}\right|} \ln \left(\frac{1}{\lambda_{n}} \ln \frac{1}{\left|g_{n}\right|}\right)}{\ln \ln \frac{1}{\left|g_{n}\right|} \ln \left(\frac{1}{\lambda_{n}} \ln \frac{1}{\left|f_{n}\right|}\right)} .
$$

If, moreover, $\kappa_{n}[F] \nearrow+\infty$ as $n_{0} \leq n \rightarrow \infty$ then

$$
\lambda_{l, l}[F]_{G}=p_{l}:=\varliminf_{n \rightarrow \infty} \frac{\ln \ln \frac{1}{\left|f_{n}\right|} \ln \left(\frac{1}{\lambda_{n}} \ln \frac{1}{\left|g_{n}\right|}\right)}{\ln \ln \frac{1}{\left|g_{n}\right|} \ln \left(\frac{1}{\lambda_{n}} \ln \frac{1}{\left|f_{n}\right|}\right)} .
$$

Proof. Since $G$ has generalized regular logarithmic growth, by Corollary $2 \varrho_{l, l}[F]_{G}=\frac{\varrho_{l}[F]}{\varrho_{l}[G]}$, $\lambda_{l, l}[F]_{G}=\frac{\lambda_{l}[F]}{\lambda_{l}[G]}$ and by Lemma 3

$$
\varrho_{l, l}[F]_{G}=\varlimsup_{n \rightarrow \infty} \ln \ln \frac{1}{\left|f_{n}\right|} / \ln \left(\frac{1}{\lambda_{n}} \ln \frac{1}{\left|f_{n}\right|}\right) \varliminf_{n \rightarrow \infty} \ln \left(\frac{1}{\lambda_{n}} \ln \frac{1}{\left|g_{n}\right|}\right) / \ln \ln \frac{1}{\left|g_{n}\right|} \leq P_{l} .
$$

On the other hand, if $P_{l}>0$ then for every $\varepsilon \in\left(0, P_{l}\right)$ there exists an increasing to $+\infty$ sequence $\left(n_{k}\right)$ of integers such that

$$
\frac{\ln \ln \frac{1}{\left|f_{n_{k}}\right|}}{\ln \left(\frac{1}{\lambda_{n_{k}}} \ln \frac{1}{\left|f_{n_{k}}\right|}\right)} \geq\left(P_{l}-\varepsilon\right) \frac{\ln \ln \frac{1}{\left|g_{n_{k}}\right|}}{\ln \left(\frac{1}{\lambda_{n_{k}}} \ln \frac{1}{\left|g_{n_{k}}\right|}\right)},
$$

i. e. by Lemma 3

$$
\begin{aligned}
& \varrho_{l}[F]=\varlimsup_{n \rightarrow \infty} \ln \ln \frac{1}{\left|f_{n}\right|} / \ln \left(\frac{1}{\lambda_{n}} \ln \frac{1}{\left|f_{n}\right|}\right) \geq\left(P_{l}-\varepsilon\right) \underline{\lim }_{n \rightarrow \infty} \ln \ln \frac{1}{\left|g_{n}\right|} / \ln \left(\frac{1}{\lambda_{n}} \ln \frac{1}{\left|g_{n}\right|}\right)= \\
& =\left(P_{l}-\varepsilon\right) \lambda_{l}[G]=\left(P_{l}-\varepsilon\right) \varrho_{l}[G],
\end{aligned}
$$

whence in view of the arbitrariness of $\varepsilon$ we get $\varrho_{l}[F]_{G} \geq P_{l}$. For $P_{l}=0$ the last inequality is obvious. Equality (10) is proved.

The proof of (11) is similar. 
The condition $\ln n=o\left(\lambda_{n} \ln \lambda_{n}\right)$ as $n \rightarrow \infty$ implies the condition $\varlimsup_{n \rightarrow \infty} \ln \ln n / \ln \lambda_{n} \leq 1$. Therefore, using Lemmas 2 and 3 it is easy to prove the following statement.

Proposition 3. If the function $G$ has regular logarithmic growth, $\ln n=o\left(\lambda_{n} \ln \lambda_{n}\right)$, $\ln \lambda_{n+1} \sim \ln \lambda_{n}$ and $\kappa_{n}[G] \nearrow+\infty$ as $n_{0} \leq n \rightarrow \infty$ then

$$
\varrho_{R, l}[F]_{G}=\varlimsup_{n \rightarrow \infty} \frac{\lambda_{n} \ln \lambda_{n} \ln \left(1 /\left|g_{n}\right|\right)}{\ln \left(1 /\left|f_{n}\right|\right) \ln \ln \left(1 /\left|g_{n}\right|\right)} .
$$

If, moreover, $\kappa_{n}[F] \nearrow+\infty$ as $n_{0} \leq n \rightarrow \infty$ then

$$
\lambda_{R, l}[F]_{G}=\varliminf_{n \rightarrow \infty} \frac{\lambda_{n} \ln \lambda_{n} \ln \left(1 /\left|g_{n}\right|\right)}{\ln \left(1 /\left|f_{n}\right|\right) \ln \ln \left(1 /\left|g_{n}\right|\right)} .
$$

Let us turn to the results about the relative growth of functions in terms of their types. For classic growth scales, we can use Lemmas 2 and 3, and for generalized orders we need such lemma.

Lemma 4. Let $\alpha \in L, \beta \in L, x \alpha^{\prime}(x)=o(1), x \beta^{\prime}(x)=O(1)$ and $\frac{d \beta^{-1}\left(c_{1}+c_{2} \alpha(x)\right)}{d \ln x}=O(1)$ as $x \rightarrow+\infty$ for each $\left.0<c_{1}, c_{2}<+\infty\right)$. If $\ln n=o\left(\lambda_{n} \beta^{-1}\left(c \alpha\left(\lambda_{n}\right)\right)\right)$ as $n \rightarrow \infty$ for each $c \in(0,+\infty)$ and $G \in S(\Lambda)$ then

$$
\varlimsup_{n \rightarrow \infty} \exp \left\{\alpha\left(\lambda_{n}\right)-\varrho_{\alpha, \beta}[G] \beta\left(\frac{1}{\lambda_{n}} \ln \frac{1}{\left|g_{n}\right|}\right)\right\}=T_{\alpha, \beta}[G] .
$$

If, moreover, $\alpha\left(\lambda_{n+1}\right)-\alpha\left(\lambda_{n}\right) \rightarrow 0$ and $\kappa_{n}[G] \nearrow+\infty$ as $n_{0} \leq n \rightarrow \infty$ then

$$
\varliminf_{n \rightarrow \infty} \exp \left\{\alpha\left(\lambda_{n}\right)-\varrho_{\alpha, \beta}[G] \beta\left(\frac{1}{\lambda_{n}} \ln \frac{1}{\left|g_{n}\right|}\right)\right\}=t_{\alpha, \beta}[G] .
$$

Proof. Put $\alpha_{1}(x)=\exp \{\alpha(x)\}$ and $\beta_{1}(x)=\exp \left\{\varrho_{\alpha, \beta}[G] \beta(x)\right\}$. Then

$$
T_{\alpha, \beta}[G]=\varlimsup_{\sigma \rightarrow+\infty} \frac{\alpha_{1}\left(\ln M_{G}(\sigma)\right)}{\beta_{1}(\sigma)}=\varrho_{\alpha_{1}, \beta_{1}}[G] .
$$

If, for example, $c>1$ then

$$
\alpha(c x)-\alpha(x)=\alpha^{\prime}(\xi)(c-1) x \leq(c-1) \xi \alpha^{\prime}(\xi)
$$

for some $\xi \in[x, c x]$ and, since $x \alpha^{\prime}(x)=o(1)$ as $x \rightarrow+\infty$, we have $\alpha(c x)-\alpha(x) \rightarrow 0$ as $x \rightarrow+\infty$, i. e. $\alpha_{1} \in L_{s i}$. Similarly, in view of condition $x \beta^{\prime}(x)=O(1)$ as $x \rightarrow+\infty$, we have $\beta((1+o(1)) x)-\beta(x)=\beta^{\prime}(\xi) o(\xi) \rightarrow 0$ as $x \rightarrow+\infty$, whence it follows that $\beta_{1} \in L^{0}$.

Since $\beta_{1}^{-1}(x)=\beta^{-1}\left((\ln x) / \varrho_{\alpha, \beta}[G]\right)$, we have

$$
\frac{d \beta_{1}^{-1}\left(c \alpha_{1}(x)\right)}{d \ln x}=\frac{d \beta^{-1}\left((\ln c+\alpha(x)) / \varrho_{\alpha, \beta}[G]\right)}{d \ln x}=\frac{d \beta^{-1}\left(c_{1}+c_{2} \alpha(x)\right)}{d \ln x}=O(1), \quad x \rightarrow+\infty .
$$

Finally, the condition $\ln n=o\left(\lambda_{n} \beta_{1}^{-1}\left(c \alpha_{1}\left(\lambda_{n}\right)\right)\right)$ as $n \rightarrow \infty$ holds if $\ln n=$ $=o\left(\lambda_{n} \beta^{-1}\left(\left(\ln c+\alpha\left(\lambda_{n}\right)\right) / \varrho_{\alpha, \beta}[G]\right)\right)$ as $n \rightarrow \infty$. But $\left(\ln c+\alpha\left(\lambda_{n}\right)\right) / \varrho_{\alpha, \beta}[G] \geq \alpha\left(\lambda_{n}\right) /\left(2 \varrho_{\alpha, \beta}[G]\right)$ for $n \geq n_{0}$. Therefore, the last condition holds if $\ln n=o\left(\lambda_{n} \beta^{-1}\left(c \alpha\left(\lambda_{n}\right)\right)\right)$ as $n \rightarrow \infty$ for $c=1 /\left(2 \varrho_{\alpha, \beta}[G]\right)$. Thus, the functions $\alpha_{1}$ and $\beta_{1}$ satisfy the conditions of Lemma 1 and in view of (8) formula (12) is proved. Also $\frac{\alpha_{1}\left(\lambda_{n+1}\right)}{\alpha_{1}\left(\lambda_{n}\right)}=\exp \left\{\alpha\left(\lambda_{n+1}\right)-\alpha\left(\lambda_{n}\right)\right\} \rightarrow 1$ as $n \rightarrow \infty$ and, therefore, by Lemma 1 formulas (13) is correct. 
Using Lemma 4, we prove the following theorem.

Theorem 4. Let $\alpha \in L, \beta \in L, \gamma \in L, x \alpha^{\prime}(x)=O(1), x \beta^{\prime}(x)=O(1), x \gamma^{\prime}(x)=o(1)$, $\frac{d \alpha^{-1}\left(c_{1}+c_{2} \gamma(x)\right)}{d \ln x}=O(1)$ and $\frac{d \beta^{-1}\left(c_{1}+c_{2} \gamma(x)\right)}{d \ln x}=O(1)$ as $x \rightarrow+\infty$ for each $0<c_{1}, c_{2}<$ $+\infty$. Suppose that $\ln n=o\left(\lambda_{n} \alpha^{-1}\left(c \gamma\left(\lambda_{n}\right)\right)\right)$ and $\ln n=o\left(\lambda_{n} \beta^{-1}\left(c \gamma\left(\lambda_{n}\right)\right)\right)$ as $n \rightarrow \infty$ for each $c \in(0,+\infty)$.

If the function $G$ has strongly regular generalized $(\gamma, \alpha)$-growth (i. e. $0<t_{\gamma, \alpha}[G]=$ $\left.T_{\gamma, \alpha}[G]<+\infty\right), \gamma\left(\lambda_{n+1}\right)-\gamma\left(\lambda_{n}\right) \rightarrow 0$ and $\kappa_{n}[G] \nearrow+\infty$ as $n_{0} \leq n \rightarrow \infty$ then

$$
\left(T_{\alpha, \beta}[F]_{G}\right)^{\varrho_{\gamma, \alpha}[G]}=Q:=\exp \left\{\varlimsup_{n \rightarrow \infty} Q_{n}(F, G)\right\},
$$

where

$$
Q_{n}(F, G)=\varrho_{\gamma, \alpha}[G] \alpha\left(\frac{1}{\lambda_{n}} \ln \frac{1}{\left|g_{n}\right|}\right)-\varrho_{\gamma, \beta}[F] \beta\left(\frac{1}{\lambda_{n}} \ln \frac{1}{\left|f_{n}\right|}\right) .
$$

If, moreover, $\kappa_{n}[F] \nearrow \infty$ as $n_{0} \leq n \rightarrow \infty$ then

$$
\left(t_{\alpha, \beta}[F]_{G}\right)^{\varrho_{\gamma, \alpha}[G]}=q:=\exp \left\{\underline{\lim }_{n \rightarrow \infty} Q_{n}(F, G)\right\} .
$$

Proof. Since the function $G$ has strongly regular generalized $(\gamma, \alpha)$-growth, by Theorem $2\left(T_{\alpha, \beta}[F]_{G}\right)^{\varrho_{\gamma, \alpha}[G]}=T_{\gamma, \beta}[F] / T_{\gamma, \alpha}[G]$ and $\left(t_{\alpha, \beta}[F]_{G}\right)^{\varrho_{\gamma, \alpha}[G]}=t_{\gamma, \beta}[F] / t_{\gamma, \alpha}[G]$. Therefore, by Lemma 4

$$
\begin{gathered}
=\varlimsup_{n \rightarrow \infty} \exp \left\{\gamma\left(\lambda_{n}\right)-\varrho_{\gamma, \beta}[F] \beta\left(\frac{1}{\lambda_{n}} \ln \frac{1}{\left|f_{n}\right|}\right)\right\} \underline{\lim _{n \rightarrow \infty}} \exp \left\{\varrho_{\gamma, \alpha}[G] \alpha\left(\frac{1}{\lambda_{n}} \ln \frac{1}{\left|g_{n}\right|}\right)-\gamma\left(\lambda_{n}\right)\right\} \leq \\
\leq \varlimsup_{n \rightarrow \infty} \exp \left\{Q_{n}(F, G)\right\}=Q
\end{gathered}
$$

and

$$
\begin{gathered}
\left.=\varliminf_{n \rightarrow \infty} \exp \left\{\gamma\left(\lambda_{n}\right)-\varrho_{\alpha, \beta}[F] \beta\left(\frac{1}{\lambda_{n}} \ln \frac{1}{\left|f_{n}\right|}\right)\right\} \varlimsup_{n \rightarrow \infty}\right)^{\varrho_{\gamma, \alpha}[G]}= \\
\geq \varliminf_{n \rightarrow \infty} \exp \left\{\varrho_{\gamma, \alpha}[G] \alpha\left(\frac{1}{\lambda_{n}} \ln \frac{1}{\left|g_{n}\right|}\right)-\gamma\left(\lambda_{n}\right)\right\} \geq
\end{gathered}
$$

On the other hand, let $Q>0$. Then for every $Q_{1} \in(0, Q)$ there exists an increasing to $\infty$ sequence $\left(n_{k}\right)$ of integers such that $\exp \left\{Q_{n_{k}}(F, G)\right\} \geq Q_{1}$, i. e.

$$
\exp \left\{\gamma\left(\lambda_{n_{k}}\right)-\varrho_{\gamma, \beta}[F] \beta\left(\frac{1}{\lambda_{n_{k}}} \ln \frac{1}{\left|f_{n_{k}}\right|}\right)\right\}>Q_{1} \exp \left\{\gamma\left(\lambda_{n_{k}}\right)-\varrho_{\gamma, \alpha}[F] \alpha\left(\frac{1}{\lambda_{n_{k}}} \ln \frac{1}{\left|g_{n_{k}}\right|}\right)\right\}
$$

and, thus,

$$
\begin{gathered}
T_{\gamma, \beta}[F]=\varlimsup_{n \rightarrow \infty} \exp \left\{\gamma\left(\lambda_{n}\right)-\varrho_{\gamma, \beta}[F] \beta\left(\frac{1}{\lambda_{n}} \ln \frac{1}{\left|f_{n}\right|}\right)\right\} \geq \\
\geq Q_{1} \varliminf_{n \rightarrow \infty} \exp \left\{\gamma\left(\lambda_{n}\right)-\varrho_{\gamma, \alpha}[F] \alpha\left(\frac{1}{\lambda_{n}} \ln \frac{1}{\left|g_{n}\right|}\right)\right\} Q_{1} t_{\gamma, \alpha}[G]=Q_{1} T_{\gamma, \alpha}[G],
\end{gathered}
$$

whence in view of the arbitrariness of $Q_{1}$ we get $T_{\alpha, \beta}[F]_{G} \geq Q$. For $Q=0$ the last inequality is obvious. The equality $\left(T_{\alpha, \beta}[F]_{G}\right)^{\varrho_{\gamma, \alpha}[G]}=Q$ is proved.

Similar we prove the inequality $t_{\alpha, \beta}[F]_{G} \leq q$, i. e. we get the equality $\left(t_{\alpha, \beta}[F]_{G}\right)^{\varrho_{\gamma, \alpha}[G]}=q$. The proof of Theorem 4 is complete. 
Next three statements are proved in general a way and we will drop their proofs. Using Corollary 2 and Lemma 2, we get the following statement.

Proposition 4. If the function $G$ has the strongly regular growth (i. e. $0<t_{R}[G]=$ $\left.=T_{R}[G]<+\infty\right)$, $\ln n=o\left(\lambda_{n}\right), \lambda_{n+1} \sim \lambda_{n}$ and $\kappa_{n}[G] \nearrow+\infty$ as $n_{0} \leq n \rightarrow \infty$ then

$$
\left(T_{R, R}[F]_{G}\right)^{\varrho_{R}[G]}=\varlimsup_{n \rightarrow+\infty} \frac{\varrho_{R}[G]}{\varrho_{R}[F]}\left|f_{n}\right|^{\varrho_{R}[F] / \lambda_{n}}\left|g_{n}\right|^{-\varrho_{R}[G] / \lambda_{n}}
$$

If, moreover, $\kappa_{n}[F] \nearrow+\infty$ as $n_{0} \leq n \rightarrow \infty$ then

$$
\left(t_{R, R}[F]_{G}\right)^{\varrho_{R}[G]}=\varliminf_{n \rightarrow+\infty} \frac{\varrho_{R}[G]}{\varrho_{R}[F]}\left|f_{n}\right|^{\varrho_{R}[F] / \lambda_{n}}\left|g_{n}\right|^{-\varrho_{R}[G] / \lambda_{n}}
$$

Since the condition $\ln \ln n=o\left(\ln \lambda_{n}\right)$ as $n \rightarrow \infty$ implies the condition $\ln n=$ $=o\left(\lambda_{n}^{\varrho /(\varrho-1)}\right)$ as $n \rightarrow \infty$ for every $\varrho>1$, using Corollary 2 and Lemma 3, we get the next statement.

Proposition 5. Let $1<\varrho_{l}[F], \varrho_{l}[G]<+\infty$. If the function $G$ has the strongly regular logarithmic growth (i. e. $0<t_{l}[G]=T_{l}[G]<+\infty$ ), $\ln \ln n=o\left(\ln \lambda_{n}\right), \lambda_{n+1} \sim \lambda_{n}$ and $\kappa_{n}[G] \nearrow+\infty$ as $n_{0} \leq n \rightarrow \infty$ then

$$
\frac{A\left(\varrho_{l}[G]\right)}{A\left(\varrho_{l}[F]\right)}\left(T_{l, l}[F]_{G}\right)^{\varrho_{R}[G]}=\varlimsup_{n \rightarrow \infty} \lambda_{n}^{\varrho_{l}[F]-\varrho_{l}[G]}\left(\ln \frac{1}{\left|f_{n}\right|}\right)^{1-\varrho_{l}[F]}\left(\ln \frac{1}{\left|g_{n}\right|}\right)^{\varrho_{l}[G]-1}
$$

If, moreover, $\kappa_{n}[F] \nearrow+\infty$ as $n_{0} \leq n \rightarrow \infty$ then

$$
\frac{A\left(\varrho_{l}[G]\right)}{A\left(\varrho_{l}[F]\right)}\left(t_{R, R}[F]_{G}\right)^{\varrho_{R}[G]}=\varliminf_{n \rightarrow \infty} \lambda_{n}^{\varrho_{l}[F]-\varrho_{l}[G]}\left(\ln \frac{1}{\left|f_{n}\right|}\right)^{1-\varrho_{l}[F]}\left(\ln \frac{1}{\left|g_{n}\right|}\right)^{\varrho_{l}[G]-1}
$$

Finally, since the condition $\ln \ln n=o\left(\ln \lambda_{n}\right)$ as $n \rightarrow \infty$ implies the condition $\ln n=o\left(\lambda_{n}\right)$ as $n \rightarrow \infty$, using Corollary 2 and Lemmas 2 and 3, we get the next statement.

Proposition 6. Let $1<\varrho_{l}[G]<+\infty$. If the function $G$ has the strongly regular logarithmic growth, $\ln \ln n=o\left(\ln \lambda_{n}\right), \lambda_{n+1} \sim \lambda_{n}$ and $\kappa_{n}[G] \nearrow+\infty$ as $n_{0} \leq n \rightarrow \infty$ then

$$
e \varrho_{R}[F] A\left(\varrho_{l}[G]\right)\left(T_{R, l}[F]_{G}\right)^{\varrho_{R}[G]}=\varlimsup_{n \rightarrow \infty} \lambda_{n}^{1-\varrho_{l}[G]}\left|f_{n}\right|^{\varrho_{l}[F] / \lambda_{n}}\left(\ln \frac{1}{\left|g_{n}\right|}\right)^{\varrho_{l}[G]-1} .
$$

If, moreover, $\kappa_{n}[F] \nearrow+\infty$ as $n_{0} \leq n \rightarrow \infty$ then

$$
e \varrho_{R}[F] A\left(\varrho_{l}[G]\right)\left(t_{R, l}[F]_{G}\right)^{\varrho_{R}[G]}=\varliminf_{n \rightarrow \infty} \lambda_{n}^{1-\varrho_{l}[G]}\left|f_{n}\right|^{\varrho_{l}[F] / \lambda_{n}}\left(\ln \frac{1}{\left|g_{n}\right|}\right)^{\varrho_{l}[G]-1} .
$$




\section{REFERENCES}

[1] Roy Ch. On the relative order and lower order of an entire functiion. Bull. Soc. Cal. Math. Soc., 2010, 102 (1), 17-26.

[2] Data S. K., Maji A. R. Relative order of entire functions in terms of their maximum terms. Int. Journal of Math. Analysis, 2011, 5 (43), 2119-2126.

[3] Data S. K., Biswas T., Ghosh Ch. Growth analysis of entire functions concerning generalized relative type and generalized relative weak type. Facta Univ.(NIS). Ser. math. inform., 2015, 30 (3), 295-324.

[4] Data S. K., Biswas T., Hoque A. Some results on the growth analysis of entire function using their maximum terms and relative $L^{*}$-order. Journ. Math. Extension, 2016, 10 (2), 59-73.

[5] Data S. K., Biswas T., Das P. Some results on generalized relative order of meromorohic functions. Ufa Math. Journ., 2016, 8 (2), 92-103.

[6] Data S. K., Biswas T. Growth analysis of entire functions of two complex variables. Sahad Communications in Math. Analysis, 2016, 3 (2), 13-22.

[7] Data S. K., Biswas T. Some growth analysis of entire functions in the form of vector valued Dirichlet series on the basis on their relative Ritt $L^{*}$-order and relative Ritt $L^{*}$-lower order. New Trends in Math. Sci., 2017, 5 (2), 97-103.

[8] Sheremeta M. M. Asymptotic behaviours of entire functions given by power series and Dirichlet series. Doct. diss., Kiev, 1987. (in Russian)

[9] Mulyava O. M., Sheremeta M. M. Relative growth of Dirichlet series. Mat. Stud., 2018, 49 (2), $158-164$.

[10] Mulyava O. M., Sheremeta M. M. Remarks to relative growth of entire Dirichlet series. Visnyk of Lviv Univ. Ser. mech.-math., 2019, 87, 73-81.

[11] Ritt J. F. On certain points in the theory of Dirichlet series. Amer. Math. J., 1928, 50, 73-83.

[12] Azpeitia A. G. A remark on the Ritt order of entire functions defined by Dirichlet series. Proc. Amer. Math. Soc., 1961, 12, 722-723.

[13] Azpeitia A.G. On the lower linear type of entire functions defined by Dirichlet series. Bull. Unione Mat. Ital., 1978, A15 (3), 635-638.

[14] Sheremeta M. M. Entire Dirichlet series. ISDO, Kyiv, 1993. (in Ukrainian)

Received 01.07.2021

Мулява О. М., Шеремета М. М. Відносне зростання иілих рядів Діріхле з різними узагальненими порядками // Буковинський матем. журнал - 2021. - Т.9, №2. - С. 22-34.

Для цілих функції $F$ і $G$, зображених рядами Діріхле зі зростаючими до $+\infty$ показниками, знайдено формули для знаходження узагальненого порядку

$$
\varrho_{\alpha, \beta}[F]_{G}=\varlimsup_{\sigma \rightarrow+\infty} \frac{\alpha\left(M_{G}^{-1}\left(M_{F}(\sigma)\right)\right)}{\beta(\sigma)}
$$

і узагальненого нижнього порядку

$$
\lambda_{\alpha, \beta}[F]_{G}=\varliminf_{\sigma \rightarrow+\infty} \frac{\alpha\left(M_{G}^{-1}\left(M_{F}(\sigma)\right)\right)}{\beta(\sigma)}
$$

функції $F$ відносно функції $G$, де $M_{F}(\sigma)=\sup \{|F(\sigma+i t)|: t \in \mathbb{R}\}$, а $\alpha$ і $\beta$ - додатні зростаючі до $+\infty$ функції.

Ключові слова і фрази: ряд Діріхле, узагальнений порядок, відносне зростання. 